\title{
SYNTHETIC APERTURE SONAR IMAGES SEGMENTATION USING DYNAMICAL MODELING ANALYSIS
}

\author{
Luis Américo Conti ${ }^{1}$ and Murilo Baptista²
}

\begin{abstract}
Symbolic Models applied to Synthetic Aperture Sonar images are proposed in order to assess the validity and reliability of use of such models and evaluate how effective they can be in terms of image classification and segmentation. We developed an approach for the description of sonar images where the pixels distribution can be transformed into points in the symbolic space in a similar way as symbolic space can encode a trajectory of a dynamical system. One of the main characteristic of approach is that points in the symbolic space are mapped respecting dynamical rules and, as a consequence, it can possible to calculate quantities that characterize the dynamical system, such as Fractal Dimension (D), Shannon Entropy $(H)$ and the amount of information of the image. It also showed potential to classify image sub-patterns based on the textural characteristics of the seabed. The proposed method reached a reasonable degree of success with results compatible with the classical techniques described in literature.
\end{abstract}

Keywords: Synthetic Aperture Sonar, image processing, dynamical models, fractal, seabed segmentation.

RESUMO. Este estudo apresenta uma proposta de metodologia para segmentação e classificação de imagens de sonar de Abertura Sintética a partir de modelos de Dinâmica Simbólica. Foram desenvolvidas, em um primeiro momento, técnicas de descrição de registros de sonar, com base na transformação da distribuição dos pixels da imagem em pontos em um espaço simbólico, codificado a partir de uma função de interação, de modo que as imagens podem ser interpretadas como sistemas dinâmicos em que trajetórias do sistema podem ser estabelecidas. Uma das características marcantes deste método é que, ao descrever uma imagem como um sistema dinâmico, é possível calcular grandezas como dimensão fractal (D) e entropia de Shannon (H) além da quantidade de informação inerente a imagem. Foi possível classificar, posteriormente, características texturais das imagens com base nas propriedades dinâmicas do espaço simbólico, o que permitiu a segmentação automática de padrões de "backscatter" indicando variações da geologia/geomorfologia do substrato marinho. 0 método proposto atingiu um razoável grau de sucesso em relação à acurácia de segmentação, com sucesso compatível com métodos alternativos descritos em literatura.

Palavras-chave: sonar de abertura sintética, processamento de imagens, modelos dinâmicos, fractal, segmentação.

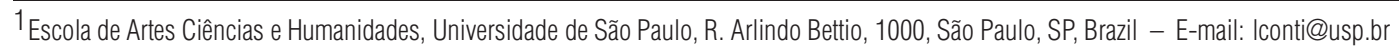

2Institute for Complex Systems and Mathematical Biology, SUPA, University of Aberdeen, United Kingdom - E-mail: murilo.baptista@abdn.ac.uk.
} 


\section{INTRODUCTION}

The increasing demand for submarine information, coupled with the rapid expansion of seafloor survey technology, has created a need for new methods of seafloor imagery processing (Reed \& Hussong, 1989). Of all remote sensing modalities available for underwater applications, acoustic methods, covering frequency ranges from a few $\mathrm{Hz}$ to several $\mathrm{MHz}$, are by far the most flexible and widely used (Capus, 2008). Thus, sonar registers revealing the geophysical characteristics of the seabed represent an essential tool for the effective knowledge of the marine environment.

Due to this growing interest, several studies have proposed methods of segmentation of sonar registers with distinct objectives. There are two basic approaches to addressing the image processing methods in sonar registers: 1) The segmentation and classification of image based on seabed texture. According to the definition of Martin et al, 2008: "segmenting" an image consists in dividing the image into homogeneous zones delimited by boundaries so as to separate the different entities in the image and "classification" consists in labeling the various components visible in an image texture (e.g. sedimentary patterns; benthic habitats). 2) feature extraction and survey underwater objects and structures such as shipwrecks, tubes, pipelines etc, and also for military purposes as called mine-like objects (MLOs).

One of the challenging issues in both approaches is the use of automatic techniques that could identify and define some characteristics of the seabed without any sort of direct human intervention. Estimations of seabed roughness and MLOs can be time consuming and often produce inconsistent results, due to the subjective nature of the analysis, automated algorithms can potentially process sonar images in a more consistent and timely manner. Several post processing techniques have been proposed in order to increase the enhancing and identification capability of classification of the seabed sonar images (Stewart, 1994; Carmichael et al., 1996; Reed et al., 2006; among others).

Most of the methods of classification is based on image processing, which uses the acoustic image of the sonar, including both echo structure and shadow shape to establish an analysis of the seabed. The image classification, also called Geospatial Bitmaps (GB) processing (Lohrenz \& Gendron, 2007) is often based on the statistical and/or their geometrical distribution of the sonar image pixels. Three basic kinds of regions can be visualized in a GB (i.e. pixel response): i. echo, ii. shadow, and iii. sea-bottom reverberation (SBR). The echo is caused by the reflection of the acoustic wave on an object while the shadow zone corresponds to a lack of acoustic reverberation behind this object. The remaining information constitutes what can be called a reverberation (Mignotte, 2000)

An usual approach to simultaneous analysis of combined texture in characterization and segmentation of patterns of response in remoted sensing images is based on autoregressive analysis. These models are based on the premise that a range of pixels in the image (2D models) exhibit certain complex behaviors similar to a quasi-periodic process in time and as such, it can be investigated by models as wavelets and the hidden Markov tree (HMT). Alternatively, we propose a dynamical approach for the description of the image where the pixels distribution in the $\mathrm{GB}$ can be transformed into points in the symbolic space in a similar way as symbolic space can encode a trajectory of a dynamical system. One of the main power of this approach is that that points in the symbolic space are mapped respecting dynamical rules and, as a consequence, it can possible to calculate quantities that characterize the dynamical system, such as Fractal Dimension (D), Shannon Entropy (H) and the amount of information of the system (or in the case, the GB), which is also related to the way points in the symbolic space are mapped to other points. All this characteristics provide a fully description of what the original image contains.

The dataset used was based on Synthetic Aperture Sonar images collected by the NATO Undersea Research Centre (NURC). The sonar platform was a MUSCLE AUV equipped with a $300 \mathrm{kHz}$ sonar with a $60 \mathrm{kHz}$ bandwidth that can achieve an along-track image resolution of approximately $3 \mathrm{~cm}$. The studied area is in the Baltic Sea off the coast of Latvia (Williams \& Coiras, 2010).

We have used a series of 9 images with different seabed patterns such as mud flat, sand rippled, rock etc. The main objective of the project was to assess the performance of a proposed classification and segmentation framework and compared it with other methods proposed on the literature.

\section{METHODOLOGY}

The creation of symbolic sequences is based on a specific encoding algorithm of a sequence of intensity levels in the sonar image (representing the echo intensity detected by the sensor). Each group with "L" pixels is codified in a symbolic sequence of length "L", with each intensity level encoded by a real number (N). The symbolic sequences were constructed using pixels along the horizontal direction of the image since it is consistent with the direction of the sonar acquisition that places the shadows along the horizontal axis (i.e. the shadow is the back part of the target). The length of the symbolic sequence $(\mathrm{L})$ should vary depending on the goal and target type and the overall characteristics of the area. 
The encoding of the sequences into real numbers need to satisfy two conditions in order to try to capture the deterministic behavior of the image:

A. Specify the closeness of two symbolic sequences, i.e. two similar symbolic sequences should be encoded by two real numbers that are close.

B. Encode the symbolic sequences in a way that a shift in two different symbolic sequences does not alter strongly the encoded sequences. And then, two nearby points of the pixel sequence representing the symbolic sequences $\mathrm{N}$ diverge according to the deterministic principles previously described.

In order to conform with conditions $A$ and $B$, the encoding of the symbolic sequences should take into consideration that symbols near the pixel $(i ; j)$ are more representative than symbols appearing far from the pixel $(i ; j)$ and that exactly equal symbolic sequences, but one appearing in the past sequence (previous pixels) and the other appearing in the future sequence (posterior pixels) should be close but not so close.

So, given a symbolic sequence $a_{-L / 2} a_{-L / 2+1} \cdots a_{-1}$. $a_{1} a_{2} \ldots a_{L / 2}$ the encoding of the past (left) symbolic sequence, $a_{-L / 2} a_{-L / 2+1} \ldots a_{-1}$, is given by

$$
\begin{gathered}
d_{k}=\sum_{i=1}^{k}\left(N-1-a_{-1}\right) \quad \bmod (N-1) \\
\delta=1-\sum_{k=1}^{L / 2} d_{k} N^{-k}
\end{gathered}
$$

and the encoding of the future (right) sequence, $a_{1} a_{2} \ldots a_{L / 2}$, is given by

$$
\begin{gathered}
c_{k}=\sum_{i=1}^{k} \bmod (N-1) \\
\gamma=\sum_{k=1}^{L / 2} c_{k} N^{-k}
\end{gathered}
$$

Usually, the encoding would be done using a $\bmod (N)$ operation. In this work, we have considered a $\bmod (\mathrm{N}-1)$ in order to artificially create the empty spaces in the symbolic spaces (where no points observed) of Figures 2 and 3. These empty spaces caused by the existence of artificially induced forbidden sequences allow us to better visually identify different patterns in the images.

The real number $(\varepsilon[0 ; 1])$ encoding the past symbolic sequence is represented by $\delta$ and the real number representing the future symbolic sequence is represented by $\gamma(\varepsilon[0 ; 1])$. The encoding proposed in Eqs. $(1,2)$ and $(3,4)$ is an extension of the encoding originally proposed to study $2 \mathrm{D}$ chaotic maps that could be encoded by an order -2 Generating Markov Partition (GPM) (Cvitanovic; 1988).

The symbolic sequence encoding can be summarized by the Figure 1.

A 2D picture showing all the points with coordinates $(\delta ; \gamma)$ is called the symbolic space of the image. Examples of symbolic spaces from a sonar image can be seen in Figure 2. Figure 2A represents the whole image codified into the symbolic space. The Figures $2 \mathrm{~B}, 2 \mathrm{C}$ and $2 \mathrm{D}$ represent the details of image in some specific seabed types, (object, flat and rippled respectively).

The symbolic space has its own dynamical characteristics. As such, all the tools, quantities, and approaches employed to characterize a dynamical system can be used for the proper characterization of the symbolic spaces. There are two quantities that are relevant for the purpose of characterizing symbolic spaces from sonar images. 1) The fractal dimension and the linear function $f(a ; b)$ from which it can be estimate the fractal dimension of the symbolic space; and 2) The average mutual information between past and future symbolic sequences. To extract the fractal dimension it is necessary to make a grid of the symbolic space $\Sigma(i ; j)$ (of a selected area in the image). This grid is constructed with boxes of sides e, as illustrated in Figure 3.

The fractal dimension of the points in the space $\Sigma(i ; j)$ is defined as

$$
D_{0}\left(\sum(i, j)\right)=\lim _{\varepsilon \rightarrow 0}-\frac{\log \left[N_{0}(\varepsilon)\right]}{\log (\varepsilon)}
$$

where $N_{0}(\varepsilon)$ refers to the number of boxes that are being occupied by points.

The linear fitting of the points with coordinates $\left[\log N_{0}(\varepsilon)-\right.$ $\log (\varepsilon)$ ] is a linear function $f(a ; b)$ with the parameters $a$ representing the constant term of the linear function and $b$ the linear coefficient reflecting the fractal dimension.

Mutual information measures the degree of uncertainty from one sequence by observing the another. Consider two sequences given by S1 (past) and S2 (future). The average mutual information between past and future sequences in the symbolic space $\Sigma(i ; j)$ (from a selected area) can be represented by MI(S1; S2). In order for MI to be large, S1 and S2 should be highly correlated. If $\mathrm{S} 1$ is either not correlated to $\mathrm{S} 2$ (or they contain few information) then MI(S1; S2) value will be small. Baptista et al. (2011) shows a simple way to calculating the mutual information between points laying in symbolic spaces using Lyapunov exponents and the fractal dimension of the symbolic space. The Lyapunov exponent measures how much nearby points spread exponentially. 

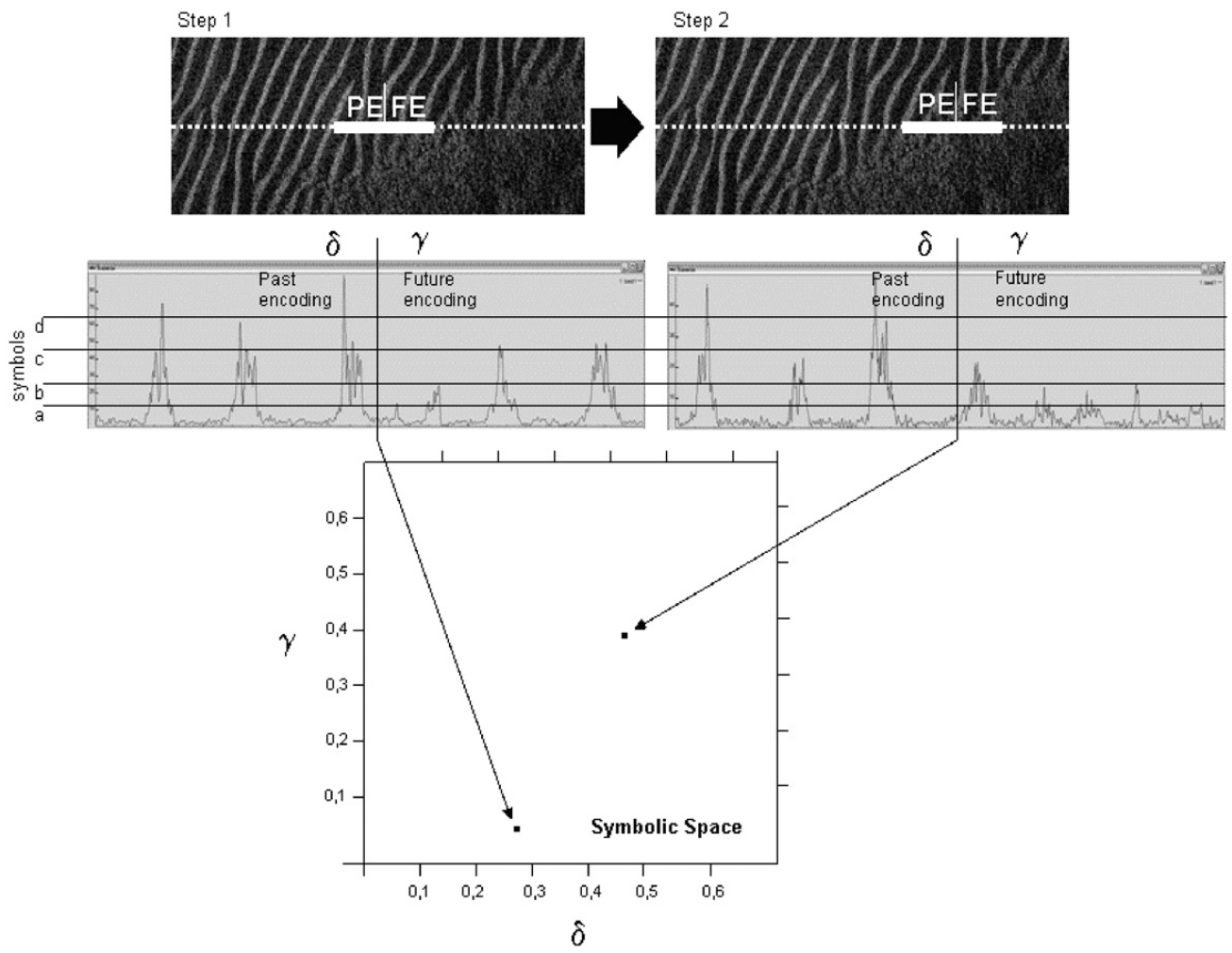

Figure 1 - Scheme of the symbolic space creation. Each pixel is encoded by a sequence of $\mathrm{N}$ pixels to its left (past encoded) and right (future encoded) given by equations [1, 2] and [3, 4]. The values of the intensity levels are reduced to N partitions or symbols ("a", "b", "c" and "d" in the figure).

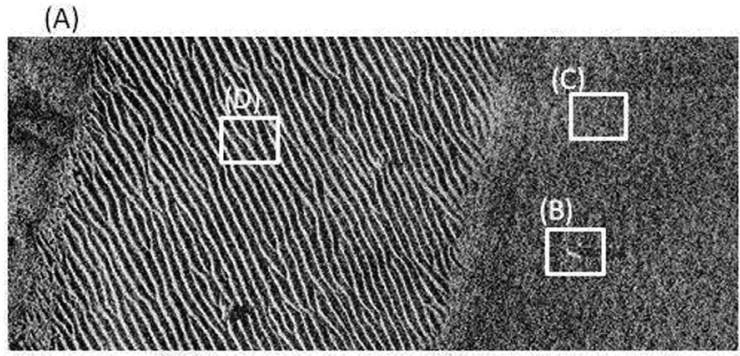

(A) (B)
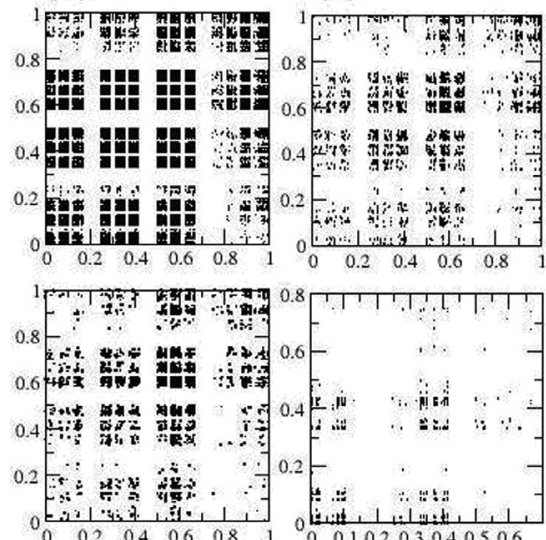

(C)

(D)

Figure 2 - Symbolic spaces of a sonar register image (A), in (B) of a selected area around a target, in (C) within the at a flat sea bed and in $(C)$ within the ripple sea bed. Selected boxes have size $L B=200$ pixels and symbolic sequences have length of $\mathrm{L}=50$. Horizontal axis represent $\delta$; (past symbolic sequences) and vertical axis represents $\gamma$ (future symbolic sequences). 


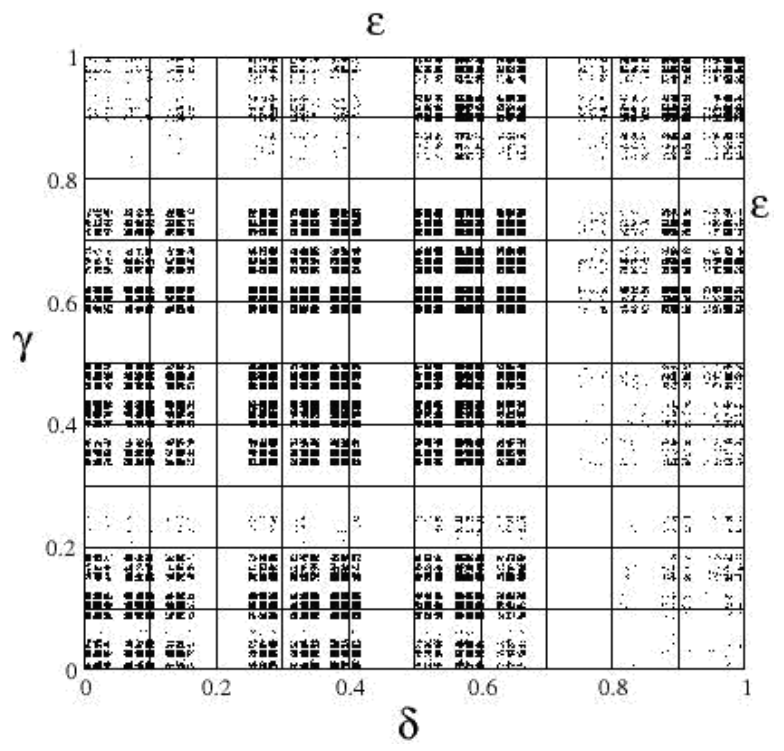

Figure 3 - Illustration of a grid of boxes of sides used to calculate the fractal dimension of the symbolic space of a selected area in the image.

The mutual information between past and future sequences should provide important characteristic to identify patterns in the image. For example, a flat sea bed, with lower signal response, produces sequences that exchange no mutual information, on the other hand, rippled seabed should exchange some information, but typically is expected that this amount is quite small, since the symbolic sequences will reproduce a repetitive periodic pattern. The mutual information between two periodic signals is zero, despite the fact that two signals are highly correlated. And we expect that the mutual information between symbolic sequences in zones with high complex seabed patterns are be large, since past and future symbolic sequences will not only be correlated but also when isolated they should be composed by many different symbols, which implies large amount of information.

\section{RESULTS AND DISCUSSION}

Analyzing different sonar images patters modulating values of $\mathrm{N}$ and $\mathrm{L}$ and the parameters $a$ and $b$ from the function given by eq. [3] it was possible to characterize several features of the sonar images and thus allowing segmenting the images based on the symbolic encoding of texture patterns. The segmentation process itself was based on a empirical characterization of these parameters in the images in specific target areas where the seabed pattern was visually identified (training). The variation of the size of the training areas also resulted in different segmentation performances.

In most of the analyzed datasets, it was possible to achieve a successful rate of identification between different types of seabed, specifically flat and rippled patterns.

We have encoded a set of 9 images and have scanned the images considering training areas of sides $B L=100$ and 50 pixels.

Figure 4 shows an example of a typical complex image with different patterns of seabed (4A) based on the segmentation process using different patterns of symbolic parameters (the colour points are given by certain values of the function $f(a ; b))$. Figure $4 \mathrm{~B}$ shows a segmentation using 9 symbols $(\mathrm{N}=9)$ with sequences of 50 pixels $(L=50)$ and training box of $100(B L=100)$ presented the better overall results to separate the flat to the rippled patters indicating the clear border between the acoustic environments. We noticed that if the scanning of the image is done with considering smaller training areas, finer details are captured. The example of the Figure 4C, shows that with smaller $L$ and BLs, specific characteristics inside the rippled pattern area can be recognized. The same happen inside of a pre-classified "flat seabed" area showed in Figure 4D.

Detailed analysis of these areas shows evidences that the symbolic sequences are identifying subtle nuances of the seabed with specific characteristics reflected in changes in the backscattering properties of the imaged and the surrounding environment. Figure 5 shows the detailed part of the top right part of the image displayed on the Figure 4 with the delimited pattern characterized by the model parameters from $4 D(N=7, L=12, B L=50)$. It is observed that the model could correctly identify darker pattern of pixels probably associated to different type of seabed composition or signal lost.

The application of the method in different images also showed that it is not clear how the parameters can be adjusted in order to determine a set of pre-defined settings to optimize the segmentation in all images. Therefore the training parameters are still crucial to segment each image. However, future studies concerning changes of parameters from the model could establish optimum adjustments to treat any sort of image dismissing the need of training making the process more automatic.

Works evolving seabed classification based on the backscattering characteristics of the bottom surface is often associated to the texture of the echoes/shadows and several of these methods of textural segmentation can be found in the literature. Most of them are based on co-occurrence matrices based on parameters such as directivity, homogeneity, contrast estimation, entropy estimation, uniformity, etc. The efficiency of such methods is quite variable, depending of the type of the analyzed surface and the quality of the register, scale etc. Table 1 shows some results of method accuracy of seabed segmentation based on different approaches. 
(a)

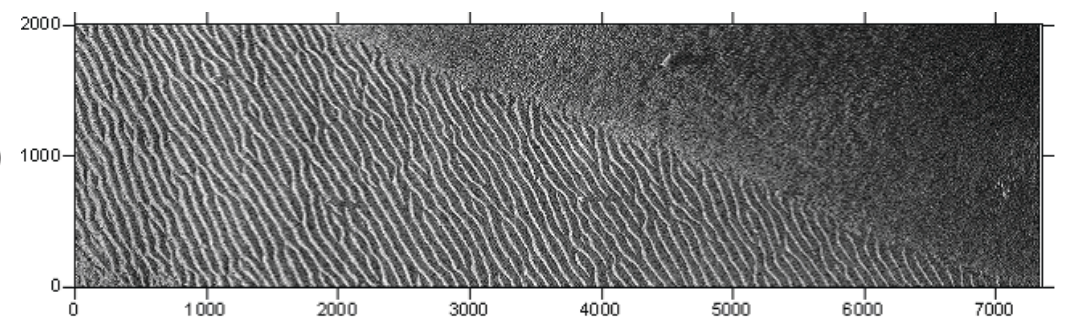

(b)

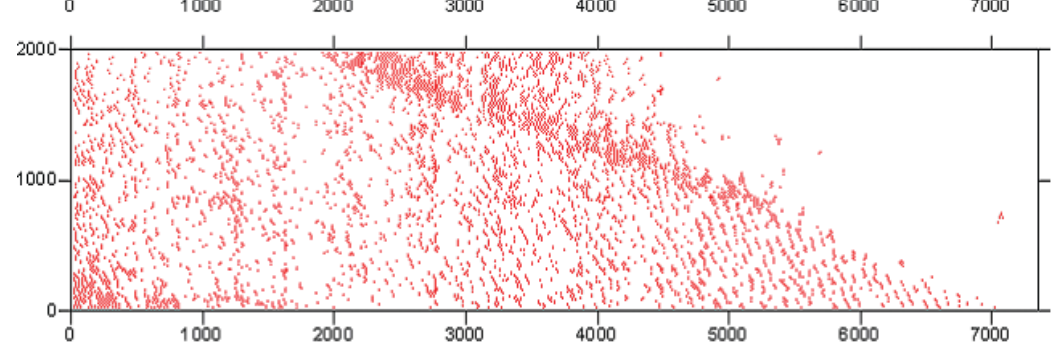

(c)
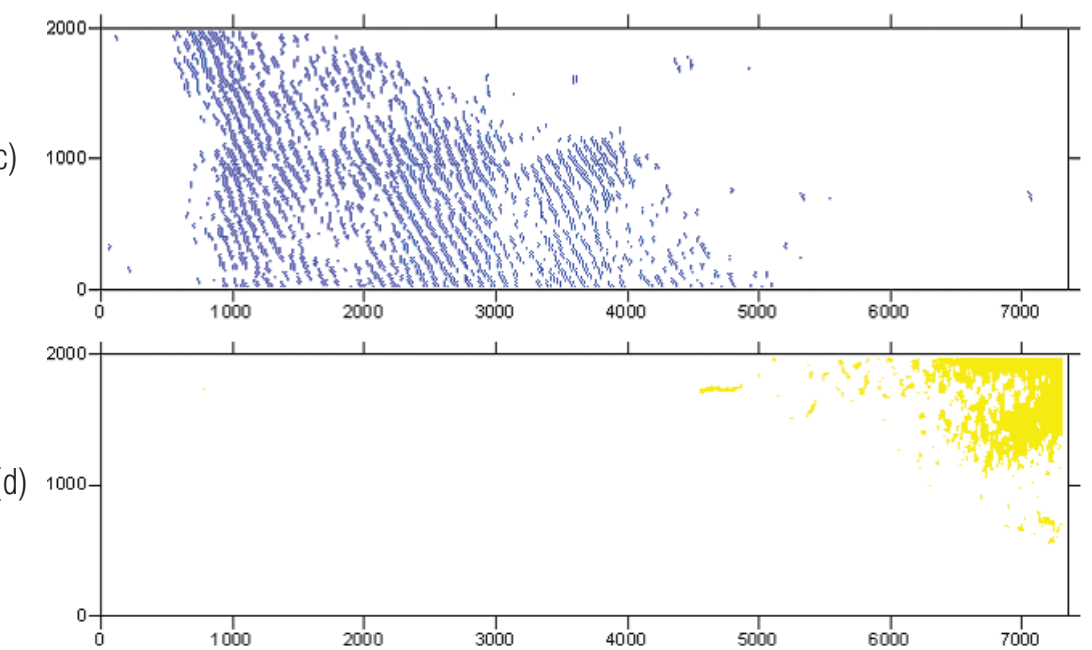

Figure 4 - Image of a sonar register (a - Top). The red dots filled representing the location of the centre of a selected sequence that has produced a value of $f(a ; b) \geq 4: 5$. The scanning of the image done with $\mathrm{BL}=100$ and $\mathrm{L}=50$. $(\mathrm{b}-$ top Centre). $B L=50$ and $L=24$ ( $c-$ bottom centre) and $B L=50$ and $L=12$ ( $d-B o t t o m)$.

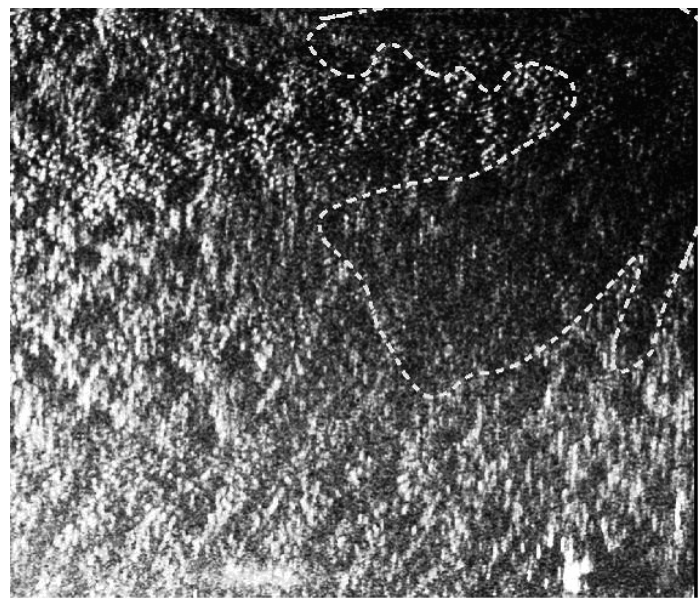

Figure $\mathbf{5}$ - Detail of a segment of the sonar image shown in Figure $4 \mathrm{~d}$. The yellow line indicates the area with lower intensity levels associated with a distinct pattern of signal reflection. 
Table 1 - Summary of results for method accuracy of seabed segmentation based on different methods.

\begin{tabular}{|c|c|c|}
\hline Author & Method & Accuracy \\
\hline Stewart et al., 1994 & Neural Networks & $\begin{array}{c}92.6 \% \text { (sediment pond) } \\
88 \% \text { (ridge flank) } \\
81 \% \text { (axial valley) }\end{array}$ \\
\hline Carmichael et al., 1996 & Multifractal & $80.69-99.37 \%$ (overall) \\
\hline Martin, 2006 & fusion of methods: weighted vote, & $87 \%$ (rock) \\
& possibility theory and & $61.3 \%$ (ripple) \\
& evidence theory. & (shadow) \\
\hline Reed et al., 2006 & power spectrum feature & $87.5 \%$ (overall) \\
\hline Lohrenz \& Gendron, 2007 & fused within a Markovian framework & and two fusion models \\
\hline Williams \& Coiras, 2010 & ripple model & $77-100 \%$ (overall) \\
\hline Conti \& Baptista, 2013 (present work) & Dynamical Model & $70-90 \%$ (overall) \\
\hline
\end{tabular}

\section{ACKNOWLEDGMENTS}

The authors would like to acknowledge the support of the UK Defense Science \& Technology Laboratory under Grant CDE17623. We would also like to acknowledge the support of NATO Under Sea Research Centre for providing a set of declassified SAS images gathered by NURC.

\section{REFERENCES}

BAPTISTA MS, RUBINGER RM, VIANA RE, SARTORELLI JC, PARLITZ $U$ \& GREBOGI C. 2012. Mutual information rate and bounds for it. Plosone, volume 7. e46745.

CAPUS CG, BANKS AC, COIRASE, TENA-RUIZI, SMITH CJ \& PETILLOT YR. 2008. Data correction for visualisation and classification of sidescan SONAR imagery ET Radar Sonar Navig., 2, 155-169p.

CARMICHAEL LM, LINNET CLARKE SJ \& CALDER BR. 1996. Seabed classification through multifractal analysis of sidescan sonar images. Proc. Inst. Electron. Eng. Radar, Sonar, and Navigation, 143: 140-148.

CVITANOVIC P, GUNARANTE GH \& PROCACCIA I. 1988. Topological and metric properties of Henon-type strange attractors. Physcal Review A, 38: 1503-1520.

MARTIN A. 2006. Comparative study of information fusion methods for sonar mages classification, International Conference on Information Fusion. Philadelphia, USA, vol. 2, 25-29 p.
MARTIN A, LAANAYA H \& ARNOLD-BOS A. 2008. Evaluation of Uncertain Image Classification and Segmentation. Journal Pattern Recognition, 39: 1987-1995.

LOHRENZ MC \& GENDRON ML. 2007. Development of an Automated Roughness Estimation Algorithm for Sidescan Imagery. In: Oceans 2007 Conference, Aberdeen, Scotland. Proceedings... June 18-21 p.

MIGNOTTE MC, COLLET C, PEREZ P \& BOUTHEMY P. 2000. Sonar Image Segmentation Using an Unsupervised Hierarchical MRF model IEEE transactions on image processing, 9, 1226-1231p.

REED TB \& HUSSONG D. 1989. Digital image processing techiques for enhancement and classification of SeaMARC 11 side scan sonar imagery. Journal of Geophyscal Research, 94: 7469-7490.

REED S, RUIZ IT, CAPUS C \& PETILLOT Y. 2006. The fusion of large scale classified side-scan sonar image mosaics. Image Processing, 15: 2049-2060.

STEWART WK, JIAN M \& MARRA M. 1994. A neural network approach to classification of side scan sonar imagery from a midocean ridge area. IEEE Journal of Oceanic Engineering, 19: 214-224.

WILLIAMS DP \& COIRAS E. 2010. On sand ripple detection in synthetic aperture sonar imagery Acoustics Speech and Signal Processing (ICASSP). In: IEEE International Conference on Issue Date, 1074$1077 \mathrm{p}$. 


\section{NOTES ABOUT THE AUTHORS}

Luis Americo Conti is Ph.D. in Marine Geology. Researcher/lecturer at the School of Arts Science and Humanities (EACH) and Center for Marine Biodiversity Research (NP-BioMar) - Universidade de São Paulo (USP). Currently is working on marine resources management focused on acoustic geophysics technologies applied on marine habitats modeling.

Murilo Baptista obtained a Ph.D. from the Universidade de São Paulo. Since 2009, is a senior lecturer at the Institute for Complex Systems and Mathematical Biology (ICSMB), University of Aberdeen. His research interest is related to applied aspects of the Theory of Information, the theory of complex networks, Ergodic Theory, network flows, dynamical systems and nonlinear systems, involving the understanding of the information transmission and collective behavior in complex and neural networks, appearance of periodic and chaotic behavior in nonlinear systems, the stability of the power-grid, capacity in flow networks, stability of cancer treatment, and social networks. 